\title{
Special nutrition in mouse developmental oocytes
}

\author{
LING YU* ${ }^{*}$ SHU-FANG WANG ${ }^{*}$ and YUAN-QING YAO \\ The General Hospital of the People's Liberation Army, Beijing 100875, P.R. China
}

Received December 15, 2011; Accepted January 30, 2012

DOI: $10.3892 / \mathrm{etm} .2012 .489$

\begin{abstract}
Investigation of nutrition-related proteins in mouse oocytes and zygotes is crucial for the development of an effective therapy for patients with infertility. Currently, we are concerned with the role of nutrition in the process of oocyte development in order to better reveal the relationship between nutrition and infertility. We collected mouse oocytes at three different developmental stages: germinal vesicle (GV) stage, metaphase II-arrested (MII) stage and fertilized oocytes (zygotes). Semi-quantitative mass spectrometry and GeneMapper software were used to analyze nutrition-related proteins in these oocytes. Various specific proteins were abundantly expressed in the mouse oocytes. These proteins included heat shock proteins and $\mathrm{Ybx} 2$. Additional proteins which exist in important metabolism pathways also demonstrated differential expression among the three stages. We identified additional nutrition elements required for oocyte development and fertilization. The present study contributed to increased understanding of nutrition in the process of oocyte development, which may enhance the efficacy of therapy for patients with infertility.
\end{abstract}

\section{Introduction}

Infertility has been considered to be a worldwide problem that affects $9-15 \%$ of the childbearing population and $55 \%$ of those affected will seek medical advice in the hope of achieving parenthood (1). The fertilization process of oocytes appears to be simple. However, multiple signaling pathways are involved in this process, and numerous factors can regulate these pathways, including various minerals, specific proteins and a multitude of nutrients. Unfortunately, little is known concerning the fertilization process of oocytes. Many relevant questions must be addressed. For example, many embryos develop well but cannot achieve implantation, and many oocytes appear healthy

Correspondence to: Professor Yuan-Qing Yao, The General Hospital of the People's Liberation Army, No. 28 Fuxing Road, Beijing 100853, P.R. China

E-mail: yaoyq1960@126.com

*Contributed equally

Key words: nutrition, oocyte, zygote, protein but are unable to be fertilized. We know that embryos from fertilization to future development require sufficient nutrition, but the specific nutrients which play an important role are yet unknown. Although numerous substances have been demonstrated to exist in oocytes (2), the roles of these substances and the relevant molecular regulatory mechanisms remain to be clarified. Additionally, with the increasing popularity of in vitro fertilization (IVF) as a method of human reproduction in recent years, a deeper understanding of the molecular composition of oocytes and their roles may help increase the IVF pregnancy rate.

During mammalian oogenesis, the oocyte undergoes two cell cycle arrests at the dictyate or germinal vesicle $(\mathrm{GV})$ stage and the metaphase II (MII) stage. The primary cellular functions of ovulated MII-arrested mammalian oocytes are to bind and fuse with a sperm, successfully reprogram and combine the two haploid genomes, and facilitate early mitotic divisions until the embryo can initiate and carry out its own molecular programs. MII oocytes have been widely used to investigate the effect of osmotic stress on the developmental competence and to perform various studies, including routine oocyte cryopreservation and the reprogramming of somatic cell nuclei (3). In contrast, oocytes at GV stage show no reprogramming activity, and zygotes exhibit low or no reprogramming activity. A comparison of the proteomes of oocytes at different developmental stages may help identify the factors responsible for fertilization and implantation.

Reproductive risk related to certain proteins and nutrients between the oocyte and zygote is a critical step in determining fertilization. Some proteins and nutrients are associated with reproductive risks, which are critical in determining fertilization. To date, most functional analyses of oocyte proteins have been limited to proteins that are primarily expressed in the oocyte. However, functional analysis of other abundant nonoocyte-restricted proteins in a developmental context would also be highly productive. In the present study, proteomes of mouse oocytes at different developmental stages were analyzed using an LTQ Orbitrap mass spectrometer. Numerous proteins identified in this study have not been previously reported, and some proteins were abundantly expressed in the oocytes at different developmental stages. These proteins may be vital nutritional elements for developing oocytes.

\section{Materials and methods}

The materials and methods for this study are the same as described previously (4). The SPF (specific pathogen-free) 


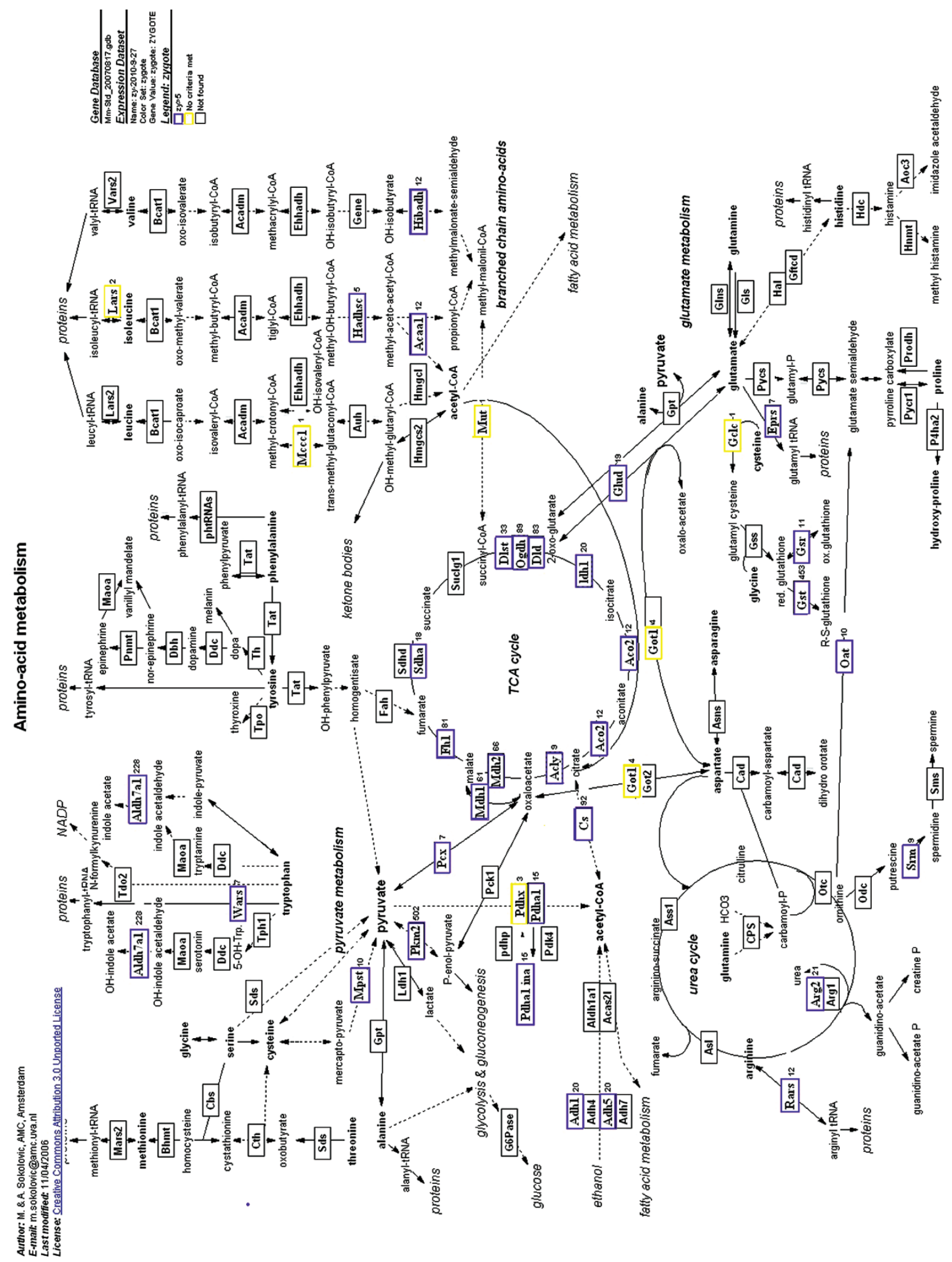

Figure 1. Highly expressed proteins involved in amino acid metabolism in zygotes. The purple rectangles represent proteins that were identified by $>5$ massspectral counts in zygotes. The yellow rectangles represent proteins that were identified by $<5$ spectrophotometry counts. 


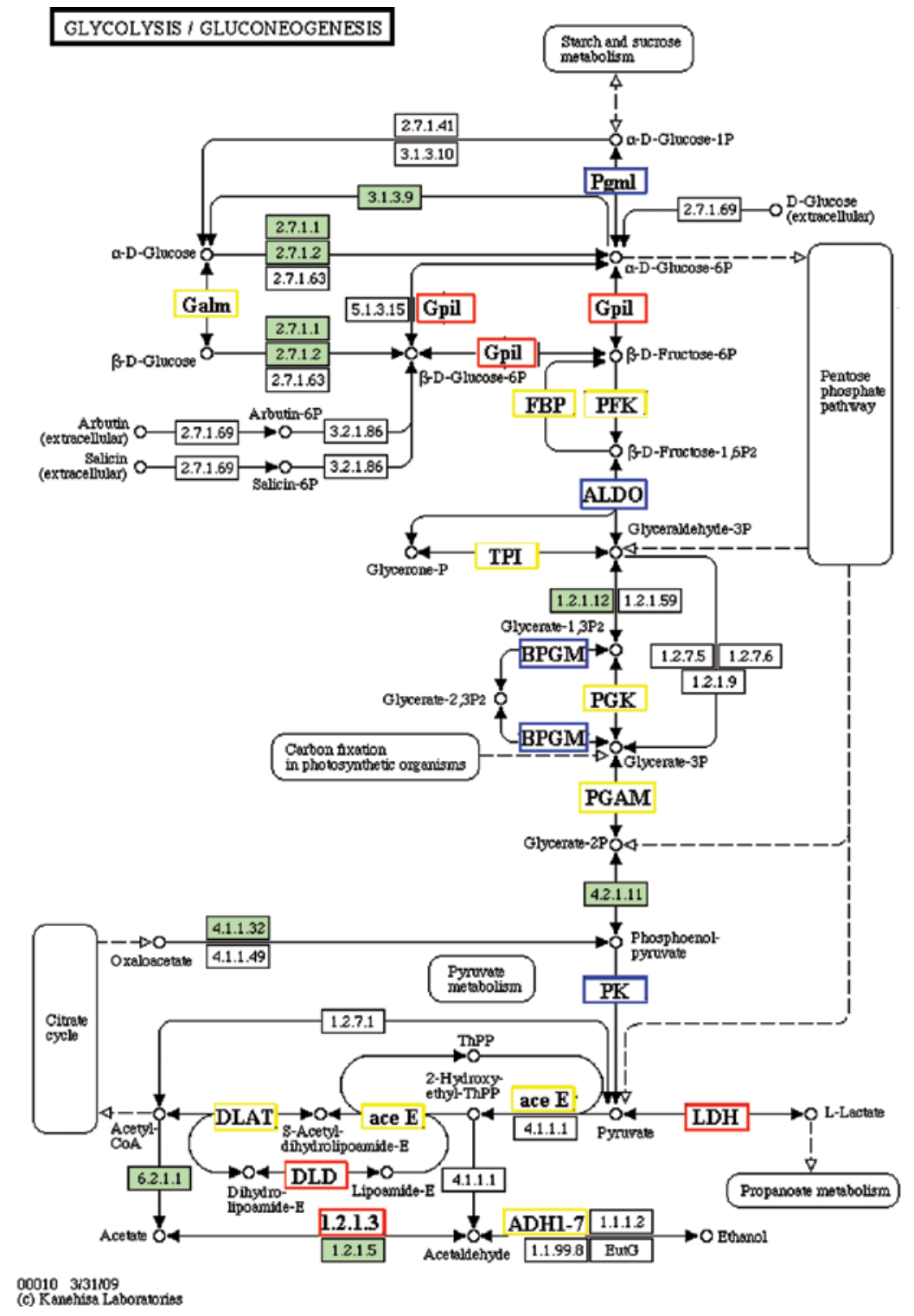

Figure 2. Expressed proteins in glycolysis or gluconeogenesis. Red rectangles represent proteins expressed more abundantly in GV oocytes compared with MII oocytes. Blue rectangles represent the proteins expressed more abundantly in MII oocytes compared with GV oocytes. Yellow rectangles represent the comparable expression level of proteins between GV and MII oocytes. Green is the color of Kyoto encyclopedia of genes and genomes (KEGG) database.

grade hybrid B6D2F1 (C57BL/6xDBA/2) mice were housed at the animal facility of the National Institute of Biological Sciences. All studies adhered to procedures consistent with the National Institute of Biological Sciences Guide for the Care and Use of Laboratory Animals.

\section{Results}

Identification of total proteins expressed in oocytes at different developmental stages. Over 7,000 oocytes were collected at each developmental stage from the mouse strain most commonly used as a recipient for proteome. From these oocytes, we successfully identified 3,892 peptides in the GV stage, 185,643 peptides in the MII oocyte and 85,369 peptides in the zygote using an LTQ Orbitrap mass spectrometer. According to this criterion, the total protein numbers identified in GV, MII and zygotes were 2,354, 2,973 and 2,082, respec- tively. Furthermore, various specific proteins, substances related to metabolism and signaling pathways were analyzed.

Ybx2. Ybx2, a member of the multifunctional Y-box protein family, is implicated to play a key role in repressing the translation of paternal mRNAs. The expression of Ybx 2 in mouse oocytes has been demonstrated previously (5). Here, the expression of Ybx 2 was detected in mouse oocytes and zygotes. The results showed that $\mathrm{Ybx} 2$ was a very abundant protein in GV oocytes, and its level gradually decreased with the development of oocytes. The expression of $\mathrm{Ybx} 2$ in zygotes was very low. The decreased expression of $\mathrm{Ybx} 2$ protein is essential for oocyte maturation and fertilization.

Heat shock-related genes. The expression of heat shock proteins (HSPs) increases when cells are exposed to elevated temperatures or other stress (6), and HSPs function as molecular 
chaperones, which play a critical role in protein folding and unfolding, intracellular trafficking of proteins and coping with proteins denatured by heat or other stresses (7). Sixteen HSPs (Hspa8, Hsp90aa1, Hspa2, Hspa4, Hspa1b, Hspa9, Trap1, Hsbp1, Hspe1, Hspa41 Isoform 1, Hspa4l Isoform 2, Hspa14 Isoform 1, Hspa14 Isoform 2, Hspd1, Ahsal and Hsph1) were found to be expressed in the different developmental stages of oocytes; most exhibited high expression in the MII oocytes, but low expression in the zygotes. Hspa8, Hsp90aal and Hspd1 were highly expressed in all three stages and their expression levels significantly decreased in the zygotes. Hspa9 was identified only in the GV stage. When oocytes develop into zygotes, oocytes consume a plentitude of nutrition previously stored in the GV and MII stage. The expression of certain HSPs had a downward trend, confirming the fact that oocytes consume HSPs in order to adapt to adverse environments.

Amino acid metabolism. Amino acids are vital nutritional elements and this dependency increases throughout the pre-implantation period (8). The presence of amino acids in maturation media increases the success rate of IVF cycles and the number of embryos that reach the blastocyst stage (9). We found that proteins involved in amino acid metabolism were highly enriched in the zygotes (Fig. 1), where they may play essential roles in the development of mammalian pre-implantation embryos. Forty proteins were identified in zygotes and 33 exhibited high expression. In amino acid metabolism, zygotes express many key factors involved in energy, including Aldh7a1 and Pkm2. Aldh7a1 was detected in mouse embryos and extraembryonic cells; it regulates energy production through altering aldehyde dehydrogenase (NAD) activity (10). Amino acids provide energy for fertilization and the developmental process of zygotes. Therefore, amino acid metabolism is critical for pregnancy.

Pyruvate metabolism. Much attention has been paid to pyruvate metabolism, since it is an important energy source for the development of oocytes. PKM2 exhibited high expression in MII oocytes. PKM2, belonging to pyruvate kinase, is considered to be a protein associated with energy metabolism (11). The fertilization of oocytes requires the involvement of energy metabolism in which PKM2 is consumed. Apart from PKM2, numerous proteins associated with pyruvate metabolism were detected in our study. Thus, pyruvate metabolism not only provides energy, but also plays an important role in the development of oocytes. However, detailed effects of pyruvate remain to be clarified.

Glycolysis or gluconeogenesis. Glycolysis is a decomposition process to provide energy; gluconeogenesis is a synthesis process to store energy. Since oocytes themselves are unable to take up L-alanine and poorly metabolize glucose for energy production, they obtain these amino acids and products of glycolysis, which are essential for their development and function (12). In our study, many proteins were detected in both pathways at all developmental stages of oocytes (Fig. 2). There are always different proteins exhibiting high expression at every stage of oocyte development. It appears that glycolysis and gluconeogenesis are both important metabolic processes in the development of oocytes.

\section{Discussion}

During oogenesis in mammals, the oocyte grows by more than two orders of magnitude, produces large quantities of a myriad of macromolecules and undergoes a complex series of morphologic and developmental changes (13). A tremendous energy toll is required for the developmental process of oocytes. Oocytes must meet their energy requirements during development by modulating a number of metabolic pathways that generate ATP. Additionally, nutrition is essential for sustaining life, and lack of various nutritional components may induce fertilization failure or embryonic dysplasia. Therefore, the relationship between nutrients and embryonic development was addressed in the present study. Compared with the methods previously used, in the present study, more accurate semi-quantitative MS proteomic analysis was employed to detect the composition of the nutrients found in oocytes and zygotes (14). The results obtained from our recent proteomic studies have provided a significant molecular insight into the processes of oocyte maturation, fertilization and pre-implantation development.

Based on the evidence obtained from previous metabolic labeling experiments and recent proteomic analysis, it appears that synthesis of these proteins begins during oocyte growth, and these factors are then stored in the egg for future utilization following fertilization (15). We found that a number of proteins were highly expressed in MII oocytes or in GV stage oocytes, but exhibited low expression in zygotes, further supporting this hypothesis. In the present study, we identified Astl that has never been associated with embryonic development. It should be noted that the oocytes stored many nutrients for further development, and the expression of Astl was increased 100 -fold in the MII oocytes when compared with that in the zygotes, indicating that Astl is a crucial factor in early embryo development.

Twelve selenium (Se)-binding proteins were detected in this study, and they were expressed in different stages. Se has been demonstrated to be an essential element for normal testicular development, spermatogenesis and spermatozoa motility, indicating its essential effect for fertility (16). It has been reported that Se deficiency lowers the reproduction rates in humans as well as in animals (17). Previous studies have shown that mouse pre-implantation embryos exposed to oxidative stress normally implant when cultured with insulin-like growth factor I and II, epidermal growth factor, insulin, transferrin and selenium (18). Therefore, 12 Se-binding proteins may be critical for the developing of oocytes. Human reproductive cells are also very sensitive to environmental changes. For example, the expression of HSPs has been reported to exhibit differential expression levels at different stages of development during spermatogenesis. Therefore, HSPs were indicated to play a significant role in the oogenesis and development of oocytes. However, more detailed studies are needed to clarify this issue. Numerous proteins involved in energy metabolism were detected, and some of these proteins were significant differentially expressed in oocytes or zygotes. These proteins found to have increased expression only in MII oocytes may be special factors for storage in the MII oocytes, in order to prepare for the period of fertilization. The fertilization of oocytes requires energy; thus energy metabolism is extremely 
important. Proteins such as, Aldh7a1, Pkm2 and Ckb that are highly expressed in zygotes may be associated with energy provisions for the development of oocytes.

In recent years, IVF has become a major treatment method for infertility when other methods of assisted reproductive technologies fail. Despite recent advances in IVF technology, many important issues associated with IVF need to be resolved. For example, low implantation rates and high multiple pregnancy rates may lead to our inability to accurately assess the reproductive potential of individual embryos. Development of non-invasive predictors is crucial to overcome the limitations of morphologic observation as assessment of the reproductive potential of an embryo. Our recent results provide the possibility of selection of the most viable embryos using amino acid and carbohydrate metabolism as predictors. In summary, our recent study provides a valuable basis and novel method for the increased understanding of the molecular mechanisms of early embryonic development and non-invasive quality assessment of embryos.

\section{Acknowledgements}

This project is supported by the China Postdoctoral Science Foundation.

\section{References}

1. Gurunath S, Pandian Z, Anderson RA and Bhattacharya S: Defining infertility - a systematic review of prevalence studies. Hum Reprod Update 17: 575-588, 2011.

2. Yurttas P, Morency E and Coonrod SA: Use of proteomics to identify highly abundant maternal factors that drive the egg-toembryo transition. Reproduction 139: 809-823, 2010.

3. Boiso I, Marti M, Santalo J, Ponsa M, Barri PN and Veiga A: A confocal microscopy analysis of the spindle and chromosome configurations of human oocytes cryopreserved at the germinal vesicle and metaphase II stage. Hum Reprod 17: 1885-1891, 2002 .
4. Wang S, Ou D, Yin J, Wu G and Wang J: Proteome of mouse oocytes at different developmental stages. Proc Natl Acad Sci USA 107: 17639-17644, 2010.

5. Yu J, Hecht NB and Schultz RM: Expression of MSY2 in mouse oocytes and preimplantation embryos. Biol Reprod 65: 1260-1270, 2001

6. De Maio A: Heat shock proteins: facts, thoughts, and dreams. Shock 11: 1-12, 1999.

7. Akerfelt M, Vihervaara A, Laiho A, Conter A, Christians ES, Sistonen L and Henriksson E: Heat shock transcription factor 1 localizes to sex chromatin during meiotic repression. J Biol Chem 285: 34469-34476, 2010.

8. Leese HJ, Sturmey RG, Baumann CG and McEvoy TG: Embryo viability and metabolism: obeying the quiet rules. Hum Reprod 22: 3047-3050, 2007.

9. Gardner DK, Lane M, Spitzer A and Batt PA: Enhanced rates of cleavage and development for sheep zygotes cultured to the blastocyst stage in vitro in the absence of serum and somatic cells: amino acids, vitamins, and culturing embryos in groups stimulate development. Biol Reprod 50: 390-400, 1994.

10. Sorolla MA, Rodriguez-Colman MJ, Tamarit J, Ortega Z, Lucas JJ, Ferrer I, Ros J and Cabiscol E: Protein oxidation in Huntington disease affects energy production and vitamin B6 metabolism. Free Radic Biol Med 49: 612-621, 2010.

11. Macintyre AN and Rathmell JC: PKM2 and the tricky balance of growth and energy in cancer. Mol Cell 42: 713-714, 2011.

12. Eppig J: Mouse oocytes control metabolic co-operativity between oocytes and cumulus cells. Reprod Fertil Dev 17: 1-2, 2005.

13. Borrell C, Rué M, I Pasarin M, Benach J and E Kunst A: The measurement of inequalities in health. Gac Sanit 14: 20-33, 2000.

14. Malmstrom J, Beck M, Schmidt A, Lange V, Deutsch EW and Aebersold R: Proteome-wide cellular protein concentrations of the human pathogen Leptospira interrogans. Nature 460: 762-765, 2009.

15. Tsujii H, Taniguchi $\mathrm{N}$ and Hamano K: Development of Mongolian gerbil embryos in chemically defined media: effects of osmolarity, glucose and phosphate. J Reprod Dev 50: 653-659, 2004.

16. Hawkes WC and Turek PJ: Effects of dietary selenium on sperm motility in healthy men. J Androl 22: 764-772, 2001.

17. Tang CC, Chen HN and Rui HF: The effects of selenium on gestation, fertility, and offspring in mice. Biol Trace Elem Res 30: 227-231, 1991.

18. Meldrum DR: Factors affecting embryo implantation after human in vitro fertilization. Am J Obstet Gynecol 165: 1896-1897, 1991. 